\title{
What Can Free Money Tell Us on the Virtual Black Market?
}

\author{
Kyungmoon Woo \\ Seoul National University \\ kwoo@popeye.snu.ac.kr \\ Hyun-chul Kim \\ Seoul National University \\ hyunchulk@gmail.com
}

Hyukmin Kwon

Korea University

hack@korea.ac.kr

\author{
Huy Kang Kim \\ Korea University \\ cenda@korea.ac.kr
}

\begin{abstract}
"Real money trading" or "Gold farming" refers to a set of illicit practices for gathering and distributing virtual goods in online games for real money. Unlike previous work, we use network-wide economic interactions among in-game characters as a lens to monitor, detect and identify gold farming networks. Our work is based on a set of real ingame trade activity logs collected for one month in year 2010 from the world's second largest MMORPG called AION (with 3.4 million subscribers). This is the first work that empirically (i) shows that "free money network" is a promising measure/approximation for detecting and characterizing gold farming networks, and (ii) measures the size of the free money net and in-game virtual economy in a large-scale MMORPG in terms of the cash flow.
\end{abstract}

\section{Categories and Subject Descriptors}

J.4 [Computer Applications]: Social and Behavioral Sciences

\section{General Terms}

Economics, Human Factors, Measurement, Security

\section{Keywords}

Online game security, Gold farming, Real money trading

\section{INTRODUCTION}

"Real money trading" or "Gold farming" refers to a set of illicit practices for gathering and distributing virtual goods in online games for real money [1]. During the last decade gold farming has become a vast enterprise (a best estimate suggests that in Asia, where most of the gold farmers dwell, more than 400,000 players spend their days stocking up on gold [2]). Gold farming industries have been grown up on the periphery of the virtual world of online games known as MMORPGs (Massively Multiplayer Online Role Playing Games) such as World of Warcraft, EverQuest, and AION.

One of the core components of the virtual world that helps to attract millions of gamers along with its fantastic settings of landscape, characters and creatures is a virtual economy. Originally, virtual goods (e.g., armor and weapons) and in-game currency are designed to be acquired only through the substantial time investments, typically

Copyright is held by the author/owner(s).

SIGCOMM'11, August 15-19, 2011, Toronto, Ontario, Canada.

ACM 978-1-4503-0797-0/11/08. from several months to even years, thus their acquisition is definitely a main goal of gamers. Yet, these goods and currencies can also be sold to or obtained from other players via trade or exchange. Trade often leads gamers with limited time for play to rather purchase virtual capital (with real money) to enjoy more exciting challenges $[1,3]$. That is where the business model (i.e., black market) of Gold farmers has been established.

Gold farming has been considered malicious by both the game companies and the player communities [1,2] due to the following reasons: (i) While in-game economies are carefully designed in a way that virtual products serve as sinks to remove money from circulation, gold farmers and buyers inject currency into the economy which creates hyper-inflationary pressure, unintended arbitrage opportunities, and other perverse incentives for market agents [1]. (ii) Farmers affect other players' experiences in a disturbing, distracting, malicious, and even illegal way, by employing anti-social computer scripts (i.e., bots) to automate the farming process, as well as often engaging in the theft of account, ID, and financial information from their customers [4]. (iii) Farming explicitly and unfairly violates the rules of play and upsets the meritocratic and fantasy-based nature of the games thus potentially driving legitimate players away [1]. For these reasons, game companies have tried to detect and ban farming accounts.

So far, the existing methods for combating gold farmers have mostly focused on distinguishing individual, automated game bots from human players using data mining techniques, Turing test based human interactive proofs (HIPs) (e.g., CAPTCHA), and human observational proofs (HOPs) [5]. These methods differentiate bots from human players by their in-game behavioral biometrics or responses to interactive (often intrusive to users) tests. The arms race between game vendors and bot developers has given birth to much more elusive and human-like bots [6] capable of avoiding and neutralizing even state-of-the art detection techniques.

Unlike previous defense methods mostly focused on individual bot behavior, we investigate network-wide economic interactions among in-game characters to detect and identify gold farming networks as a whole, not individual automated bots comprising (only) a part of those networks. In particular, as an initial step pursuing that direction we first show empirically that "free money network" is a light-weight, promising vehicle for detecting 


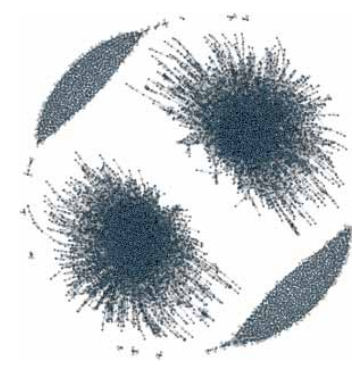

(a) Goods trading network

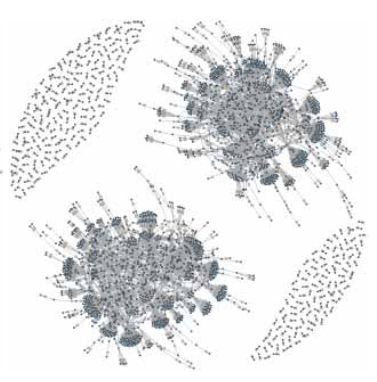

(b) Free money net only
Figure 1: AION Trade Networks.

and characterizing gold farmers. We also measure the size of the free money network as well as in-game virtual economy in terms of the cash flow based on a vast amount of real data. We use a set of (anonymized) in-game trade activity logs of AION, collected for one month from one of 41 independent yet identical game worlds (servers) in 2010 .

\section{PRELIMINARY RESULTS}

"Trade" in MMORPGs means bartering or buying goods with in-game money. Our conjecture is that free money trade activities which only give in-game money without getting any goods/items in return are likely to be either free gifts among friends or somehow related with real money trading of gold farmers. Fig. 1(a) visualizes all the trade activities logged in our dataset where nodes and edges represent characters and trade activities among them, respectively. Fig. 1(b) is a subgraph of 1(a) consists of free gift edges and related nodes only. Note that in the AION community there are two character tribes (heavenly vs. diabolic) who cannot communicate with each other, thus each tribe forms its own trade clusters.

To our surprise, while the free money network involves with only $9.7 \%(2,884 / 29,612)$ and $1.9 \%(4,719 / 252,859)$ of the nodes and edges (i.e., transactions) in the whole trade network, it takes account of $62.2 \%$ of the total transaction money during the observed period $(\$ 62,526$ out of total $\$ 100,593$ when converted to USD). We also observe that Fig. 1(b), in particular the two central clusters, clearly unveils nodes whose interaction graphs look very similar to the typical cone-shaped N-to-1 and/or 1-to-N interaction patterns of gold farmers [1] (see Fig. 2(b)). Gold farming networks mainly consists of (i) gold producers who repeatedly send free money to only a small number of designated players (i.e., brokers), (ii) brokers who collect lots of free money from many gold producers and then transfer them to buyers at no charge back, and (iii) a lot of buyers receiving free money from the brokers. This 3-tier structure shown in Fig. 2(b) naturally comes from the survival strategy of gold farmers. Brokers intervene between gold producers and buyers to maximize farming efficiency and to establish the secrecy of their network. Indirect transactions also hide the identities of high-level gold producers/bots that are, raised with substantial investments of time, the most valuable assets for gold farmers.

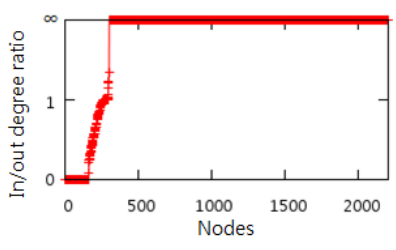

(a) In/out degree ratios

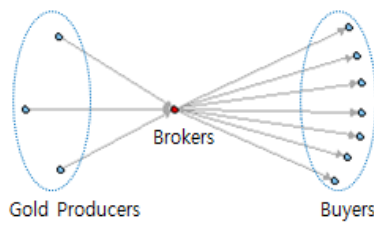

(b) A typical 3-tier shape
Figure 2: In/out degree ratios and a typical 3-tier shape.

Fig. 2(a) plots the distribution of the ratio of the number of incoming edges to that of outgoing edges for the 2,205 nodes forming the two larger central clusters in Fig. 1(b). Interestingly, we observe that $97.1 \%$ of all the nodes have either incoming or outgoing edges only. $10.8 \%$ and $86.3 \%$ of the nodes have either outgoing or incoming edges only, respectively. These uni-directional edges are the typical network-wide interaction patterns of gold producers and buyers shown in Fig. 2(b). The remaining $2.9 \%$ of the nodes mostly have a lot more number of outgoing edges (to buyers) than incoming edges (from producers), which is also the typical interaction pattern of gold brokers.

To summarize, we have found that (i) $62.2 \%$ of the total in-game cash flow was of free money, and (ii) $93.4 \%$ of the free money is highly likely to be related/connected with real money trading, i.e., virtual black market. Our current on-going work includes: (1) quantifying accuracy of the proposed approach based on the ground truth information (e.g., banned accounts), (2) finding out how many new gold farmers our approach can detect hitherto impossible, (3) further in-depth characterization of gold farmer networks, expanding our focus onto more diverse in-game social relationships as well, and (4) comparison of the revealed characteristics with those of other in-game normal users as well as real-world crime networks like drug trafficking [1] or money laundering ones.

\section{ACKNOWLEDGMENTS}

This work was supported by NAP of Korea Research Council of Fundamental Science and Technology and the ITRC support program (NIPA-2011-C1090-1111-0004) of MKE/NIPA.

\section{REFERENCES}

[1] B. Keegan et al., "Dark Gold: Statistical Properties of Clandestine Networks in Massively Multiplayer Online Games," IEEE SocialCom, 2010.

[2] R. Heeks, "Real Money from Virtual Worlds," Scientific American, 302(1), Jan. 2010.

[3] E. Castronova, Synthetic Worlds: The Business and Culture of Online Games, University of Chicago Press, 2005.

[4] G. Lastowka, "ID theft, RMT, \& Lineage," TerraNova, 2006; http://terranova.blogs.com/terra_nova/2006/07/id_theft_rmt_ nc.html $\backslash$

[5] S. Gianvecchio et al., "Battle of Botcraft: Fighting Bots in Online Games with Human Observational Proofs," ACM CCS, 2009.

[6] S. Hill, MMO Subscriber Populations, http://www. brighthub.com/video-games/mmo/articles/ 35992.aspx 